\title{
Evaluation of complement regulatory components in patients with atypical hemolytic uremic syndrome
}

\author{
HOSSEINI SOUDABEH ${ }^{1,2}, K_{A L A N T A R}$ EBRAHIM ${ }^{2,3}$, HOOMAN NAKISA ${ }^{4}$, DORGALALEH AKBAR \\ HOSSEINI SHAMSABADI ROZITA* ${ }^{4}$ TAREGH BAMEDI ${ }^{6}$
}

${ }^{1}$ Hematology and Flow Cytometry Laboratory, Ali Asghar Children Hospital, Iran University of Medical Sciences, Tehran, Iran ${ }^{2}$ Laboratory Director, Gholhak Clinical Laboratory Tehran, Iran ${ }^{3}$ Department of Immunology, School of Allied Health Science, Iran University of Medical Sciences, Tehran, Iran ${ }^{4}$ Paediatric Nephrology Ward, Ali Asghar Children Hospital, Iran University of Medical Sciences, Tehran, Iran ${ }^{5}$ Department of Hematology, School of Allied Health Science, Iran University of Medical Sciences, Tehran, Iran ${ }^{6}$ Infection Diseases and Tropical Medicine Research Center, Zahedan University of Medical Sciences, Zahedan, Iran

\begin{abstract}
Background: Atypical hemolytic uremic syndrome (aHUS), a rare disorder characterized by thrombocytopenia, microangiopathic hemolytic anemia, and acute renal failure, is associated with mutations and polymorphisms in various components and regulators of the complement alternative pathway (AP), including factor $H$, factor I, membrane cofactor protein (MCP or CD46) and factor B. This impaired regulation of the alternative pathway leads to a procoagulant state with microthrombi formation in the renal vasculature, which influences disease onset and progression.

Aim of the study: To evaluate the role of complement regulatory factors in occurrence of aHUS; we also included evaluation of ADAMTS13 activity and autoantibody against ADAMTS13 in order to exclude thrombotic thrombocytopenic purpura (TTP) cases, which might have overlapping clinical and laboratory findings.

Material and methods: This study was conducted on 273 individuals with aHUS. Diagnosis was based on clinical manifestations, kidney function tests, red blood cell count, morphology and reticulocyte count. Then, the ADAMTS13 autoantibody and activity and also complement factor $B$, complement factor $\mathrm{H}(\mathrm{CFH})$ and complement factor-I (CIF) were analyzed. Finally, the statistical analysis was performed by SPSS software.

Results: The mean age of our patients was 27.3 years, $55 \%$ were female and $45 \%$ were male. The mean levels of urea and creatinine concentration were $92.9 \mathrm{mg} / \mathrm{dl}$ and $5.1 \mathrm{mg} / \mathrm{dl}$, respectively. The mean levels of RBC count, Hb and HCT in these patients were lower than normal but the mean percentage of reticulocyte count was higher than normal (2.5\%). The assessment of complement regulatory factors revealed that the $B$ and $H$ factors levels were normal except in two cases but the level of factor I was higher than normal.

Conclusions: According to the results of this study, it seems that up regulation of factor I had a significant role in occurrence of aHUS in our study group.
\end{abstract}

Key words: atypical hemolytic uremic syndrome, ADAMTS 13, ADAMTS autoantibody, factor I.

(Centr Eur J Immunol 2014; 39 (1): 67-70)

\section{Introduction}

The hemolytic uremic syndrome (HUS) is a heterogeneous group of disorders characterized by a triad of thrombocytopenia, microangiopathic hemolytic anemia and acute renal failure [1]. Typical HUS occurs in infants and young children and is triggered by shiga toxin (Stx), which is a powerful exotoxin produced by some strains of bacteria such as 0157/H7 Escherichia coli and has a favorable outcome. In contrast, atypical HUS (aHUS) is a rare disease with an estimated incidence of 2 per million in some populations ( $10 \%$ of total HUS), which occurs at any age, from the neonatal period to adulthood and has a poor prognosis that ultimately leads to mortality in $25 \%$ and renal replacement therapy in $50 \%$ of cases in acute phase $[1,2]$.

Correspondence: Hosseini Soudabeh, Ali Asghar Children Hospital, Iran University of Medical Sciences, Tehran, Iran, e-mail: hosseini.soudabeh@gmail.com 
Atypical HUS is associated with dysregulation of the complement alternative pathway (AP). In half of the cases, mutations of the complement regulatory genes including factor $\mathrm{H}(\mathrm{CFH})$, membrane cofactor protein (MCP or CD46) and factor I (IF) lead to aHUS development. Mutations of $\mathrm{CFH}$ are the most frequent and account for $30-40 \%$ of all mutations in aHUS patients. These mutations result in altered binding of factor $\mathrm{H}$ to renal microvasculature and cause activation of the AP and therefore endothelial damage via complement dysregulation. And finally these events contribute to thrombotic microangiopathic anemia (TMA) and aHUS [3, 4]. The microangiopathic nature of the disease leads to an increased number of schistocytes in peripheral blood smear and high percentage of reticulocytes due to increased hemolysis. In addition to these mutations, an autoantibody against factor $\mathrm{H}$ is also thought to be linked to the disease predisposition. The anti-CFH antibody inhibits the binding of CFH to C3b and surface of the cell membrane. Mutations in MCP also account for $13 \%$ of all mutations in aHUS and leads to low $\mathrm{C} 3 \mathrm{~b}$ binding capacity and reduced cofactor activity. In contrast to $\mathrm{CFH}$ or MCP mutations, IF mutations are less common and cause a defect in secretion of this protein or disrupt the cofactor activity of IF, with modified degradation of $\mathrm{C} 3 \mathrm{~b} / \mathrm{C} 4 \mathrm{~b}$ in both fluid phase and on the surfaces. In addition to these genetic defects, mutations of factor B and complement $\mathrm{C} 3$ component have also been reported $[1,3,5]$.

Complement - aHUS must be differentiated from other TMA including thrombotic thrombocytopenic purpura (TTP) and other forms of HUS. Thrombotic thrombocytopenic purpura and aHUS have different clinical manifestations, with a predominant neurological involvement and renal involvement in TTP and HUS, respectively. Symptoms in TTP and HUS may overlap and whenever the ADAMTS13 deficiency in TTP is not detected, HUS has to be suspected. The ADAMTS13 activity below $10 \%$ of normal range is indicative of TTP. In addition, because the association of CFH mutation with the deficiency of ADAMTS13 has been reported, in several studies the determination of ADAMTS13 activity is suggested [6].

The purpose of the present study is to evaluate the status of complement regulatory proteins and complement components, ADAMTS13 and ADAMTS13 autoantibody in patients with aHUS presentation.

\section{Material and methods}

\section{Patients, including and excluding criteria}

The subjects in this experimental study were 273 patients attending departments of pediatric nephrology of Ali Asghar Children's Hospital in Tehran; and adult nephrology departments from different university hospitals in Iran between 2010 and 2013 .
Patients with the following criteria were included in the study: 1) negative history of drug-associated disease; 2) no criteria for Shiga toxin-HUS (stool culture, serology for anti-lipopolysaccharides antibodies); 3) no history of vasculitis; 4) no history of infection; and 5) ADAMTS13 activity above $10 \%$ of normal range.

Blood obtained from patients was stored in sodium citrate $(3.2 \%)$ anticoagulant for plasma separation and in plane tubes without anticoagulant for serum separation. Then, plasma and serum were separated by high-speed centrifugation $(2500 \times \mathrm{g})$.

Since all information was anonymously collected (no individual patient identifiers) and each patient name was replaced with a code, neither informed consent nor formal ethics committee approval was required. Analysis of ADAMTS13 autoantibody and protein was performed by ADAMTS13 autoantibody IMUBIND Elisa kit (American Diagnostica Inc., Stamford, CT, USA) and IMUBIND ADAMTS13 assay Elisa kit (American Diagnostica Inc., Stamford, CT, USA), respectively. Analysis of human factor $\mathrm{B}$, factor $\mathrm{H}$ and factor I were done by Binding Site radial immunodiffusion kits (In Vitro Technologies, Australia) and factor B, H, I assay kits, respectively. CBC test was performed by Mindray cell counter (5800, China), reticulocyte percentage was performed by manual vital staining by brilliant cresyl blue and percentage of schistocytes was counted on peripheral blood smear stained with Romanowsky stains.

\section{Biochemical laboratory tests}

Serum specimens were evaluated for creatinine and uric acid (ElitechGroup, France) and by Mindray Chemistry Analyzer (BS-200, China).

\section{Statistical analysis}

Obtained data were analyzed by SPSS software. $T$ test was used for comparison between two groups of males and females in this study. Results were reported as a mean \pm standard deviation (SD), minimum and maximum values for quantitative variables and percentages of categorical variables. Statistical significance was based on two-sided design-based tests evaluated at the 0.05 level of significance.

\section{Results}

The mean age of patients was 27.3 years ( 1 to 87 years), $150(55 \%)$ were males and $123(45 \%)$ were females. The mean levels of urea and creatinine concentration among aHUS patients were $92.9 \mathrm{mg} / \mathrm{dl}$ and $5.1 \mathrm{mg} / \mathrm{dl}$, respectively.

The hematological analysis revealed that the mean levels of RBC count, Hb and HCT in these patients were lower than normal and microscopic examination of peripheral blood smears showed a considerable number of schisto- 
Table 1. Hematological parameters in patients with aHUS

\begin{tabular}{|c|c|c|c|c|c|c|}
\hline \multirow[t]{2}{*}{ Index } & \multirow{2}{*}{$\begin{array}{l}\text { Normal range } \\
\left.\text { (children }{ }^{1}\right)\end{array}$} & \multirow{2}{*}{$\begin{array}{l}\text { Normal range } \\
\quad \text { (adult) }\end{array}$} & \multicolumn{4}{|c|}{ Patients } \\
\hline & & & Minimum & Maximum & Mean & SD \\
\hline $\mathrm{RBC}(\mathrm{mil} / \mu \mathrm{l})$ & $3.1-5.9$ & $\begin{array}{l}\text { (male: } 4.2-5.9 \text { ) } \\
\text { (female: } 4.1-5.1 \text { ) }\end{array}$ & 1.5 & 9.5 & 3.7 & 2.7 \\
\hline $\mathrm{Hb}(\mathrm{g} / \mathrm{dl})$ & $9.4-18.5$ & $\begin{array}{c}\text { (male: } 14-17.5 \text { ) } \\
\text { (female: } 12.3-15.3 \text { ) }\end{array}$ & 5.7 & 15.9 & 9.6 & 2.4 \\
\hline $\mathrm{HCT}(\%)$ & $28-60$ & $\begin{array}{c}\text { (male: } 41.5-50.4 \text { ) } \\
\text { (female: } 35.9-44.6)\end{array}$ & 13.8 & 51.6 & 47.2 & 5.7 \\
\hline RDW (\%) & $11.6-14.6$ & $11.6-14.6$ & 12.8 & 26.2 & 17.4 & 5.6 \\
\hline Platelets $\left(\times 10^{3} / \mu \mathrm{l}\right)$ & $84-478$ & $150-450$ & 14 & 509 & 132 & 118 \\
\hline Reticulocytes (\%) & $3-6$ & $0.5-1.5$ & 0.2 & 15 & 2.5 & 5.5 \\
\hline Schistocytes & $0-0.2$ & $0-0.2$ & 1 & 3.5 & 1.5 & 2.1 \\
\hline $\operatorname{WBC}\left(\times 10^{3} / \mu \mathrm{l}\right)$ & $4.5-17.5$ & $4.4-11.3$ & 1.8 & 28 & 10.6 & 8.7 \\
\hline
\end{tabular}

${ }^{{ }^{I}}$ Children aged $0-18$ years

Table 2. Profile of complement components and regulatory proteins in patients with aHUS

\begin{tabular}{|c|c|c|c|c|c|c|}
\hline & \multirow[t]{2}{*}{ Sex } & \multirow[t]{2}{*}{ Normal range } & \multicolumn{3}{|c|}{ Patients } & \multirow[t]{2}{*}{$p$ value } \\
\hline & & & Number & Mean & SD & \\
\hline \multirow[t]{2}{*}{$\mathbf{H}(\mathbf{m g} / \mathbf{l})$} & men & $350-590$ & 123 & 478 & 145 & \\
\hline & women & & 150 & 498 & 144 & 0.5 \\
\hline \multirow[t]{2}{*}{ I (mg/l) } & men & & 123 & 63 & 54 & \\
\hline & women & $38-58$ & 150 & 91 & 34 & 0.04 \\
\hline \multirow[t]{2}{*}{ B (mg/dl) } & men & & 123 & 322 & 96 & \\
\hline & women & $205-400$ & 150 & 312 & 73 & 0.05 \\
\hline \multirow[t]{2}{*}{$\operatorname{ADAMTS13(ng/ml)~}$} & men & $630-850$ & 123 & 446 & 221 & \\
\hline & women & & 150 & 426 & 237 & 0.6 \\
\hline \multirow[t]{2}{*}{ Anti-ADAMTS13 (Au/ml) } & men & & 123 & 26.3 & 37 & \\
\hline & women & $<40$ & 150 & 28 & 38 & 0.7 \\
\hline
\end{tabular}

cytes. The mean percentage of reticulocyte count in these patients was also higher than normal (Table 1).

Assessment of complement components and regulatory proteins of these patients revealed that the mean level of complement components, including C3 $(5.84 \mathrm{mg} / \mathrm{ml})$ and $\mathrm{C} 4(0.3 \mathrm{mg} / \mathrm{ml})$ were within normal range. The comparison between men and women revealed no statistically significant difference $(p>0.05)$. In studied patients, the means of $\mathrm{H}$ and $\mathrm{B}$ factors were within normal range.

In our patients with aHUS, factor I analysis showed a higher level than normal range and this abnormality in female patients was more obvious than in male patients with a statistically significant difference $(p=0.04)$ (Table 2$)$.

The comparison between male and female levels of ADAMTS13 and Anti-ADAMTS13 revealed no statisti- cally significant difference $(p=0.6$ and $p=0.7$, respectively) either.

\section{Discussion}

Atypical HUS is a rare disorder that occurs at any age and is associated with dysregulation of complement alternative pathway (AP). About $50 \%$ of aHUS cases occur due to mutations in complement regulatory genes including complement factor $\mathrm{H}(\mathrm{CFH})$, membrane cofactor protein (MCP or CD46) and I factor (IF). In addition to these genetic defects, mutations of factor $\mathrm{B}$ and $\mathrm{C} 3$-component have also been reported. This disorder must be differentiated from other diseases, like TMA (thrombotic microangiopathy), and TTP, by evaluation of ADAMTS13 activity. 
We found that RBC indices including RBC count, $\mathrm{Hb}$ and HCT levels were lower than normal ranges and the reticulocyte percentage was higher than normal, which showed the hemolytic aspect of disease.

A relatively high percentage of schistocytes in microscopic examination of peripheral blood smear and thrombocytopenia showed the microangiopathic nature of the disease in aHUS patients.

The evaluation of complement components in these patients revealed that $\mathrm{C} 3$ and $\mathrm{C} 4$ levels were normal and comparison between male and female patients showed no statistically significant difference $(p>0.05)$. The assessment of $\mathrm{B}$ and $\mathrm{H}$ factors revealed that these factors also were in normal ranges and comparison between two genders did not show any statistically significant difference for these complement regulatory factors $(p=0.05)$. But among our patients, we had a patient with a decreased level of factor B who presented with multiple oral abscesses; we also had a case with CFH deficiency.

Furthermore, our study revealed that the level of factor I was higher than normal in both genders and the difference between male and female was statistically significant $(p=0.04)$.

Previously, it was revealed that mutation in factor I has been demonstrated in 4-10\% of patients with aHUS and mutation in this regulatory protein is a less common cause of aHUS than CFH, or MCP mutations [6].

About $40 \%$ of mutations in factor I gene result in aHUS with a low level of factor I, but in our study there was an increased level of factor I. This is not a usual finding and requires further more precise studies, especially molecular analysis of mutations in this factor to detect any probable molecular defect that may result in an increased level of factor I and predisposition to aHUS [1].

Since half of factor I-aHUS cases progresses to endstage renal failure, it seems that early diagnosis, regular follow up, and appropriate management of these patients is of major importance.

\section{Acknowledgment}

We dedicate this article to our compassionate mentor Dr Hassan Otukesh, head of pediatric nephrology ward in Ali Asghar Children Hospital who taught us how to love and care for children in need and pain.

We also gratefully acknowledge the colleagues who participated in this study from different university hospitals in Iran and staff in Gholhak Clinical Laboratory who performed the laboratory analysis.

Authors declare no conflict of interest.

\section{References}

1. Kavanagh D, Goodship TH, Richards A (2006): Atypical haemolytic uraemic syndrome. Br Med Bull 77: 5-22.

2. Nester CM, Thomas CP (2012): Atypical hemolytic uremic syndrome: what is it, how is it diagnosed, and how is it treated? Hematology Am Soc Hematol Educ Program 2012: 617625.

3. Sellier-Leclerc AL, Fremeaux-Bacchi V, Dragon-Durey MA, et al. (2007): Differential impact of complement mutations on clinical characteristics in atypical hemolytic uremic syndrome. J Am Soc Nephrol 18: 2392-2400.

4. Noris M, Remuzzi G (2009): Atypical hemolytic-uremic syndrome. N Engl J Med 361: 1676-1687.

5. Zipfel PF, Skerka C (2006): Complement dysfunction in hemolytic uremic syndrome. Curr Opin Rheumatol 2006; 18 : 548-555.

6. Loirat C, Frémeaux-Bacchi V (2011): Atypical hemolytic uremic syndrome. Orphanet J Rare Dis 2011; 6: 60. 